\title{
First axion dark matter search with toroidal geometry
}

\author{
J. Choi* \\ Center for Axion and Precision Physics Research (CAPP), \\ Institute for Basic Science (IBS), Daejeon 34141, Republic of Korea \\ E-mail: jihooneibs.re.kr
}

We, the Institute for Basic Science Center for Axion and Precision Physics Research report the first axion dark matter search with toroidal geometry. In this pioneering search, we exclude the axion-photon coupling $g_{a \gamma \gamma}$ down to about $5 \times 10^{-8} \mathrm{GeV}^{-1}$ over the axion mass range from 24.7 to $29.1 \mu \mathrm{eV}$ at the $95 \%$ confidence level.

The European Physical Society Conference on High Energy Physics

5-12 July, 2017

Venice

${ }^{*}$ Speaker. 


\section{Introduction}

More than $80 \%$ of the content in the Universe is believed to be cold dark matter (CDM) and dark energy according to precision cosmological measurements [1]. One of the most compelling candidates for CDM is the axion [2] provided its mass is above $1 \mu \mathrm{eV}$ [3] and below $3 \mathrm{meV}$ [4]. The axion search method proposed by Sikivie [5], also known as the axion haloscope search, involves a microwave resonant cavity with a static magnetic field that induces axion conversions into microwave photons. The present state of the axion haloscope employs a cylindrical resonant cavity in a solenoidal field. But we introduced toroidal resonant cavity for axion search instead of cylindrical cavity. A toroidal geometry offers several advantages, two of which are a large volume for a given space and greatly reduced fringe fields which interfere with our amplifiers. In this pioneering search, we exclude the axion-photon coupling $g_{a \gamma \gamma}$ down to about $5 \times 10^{-8} \mathrm{GeV}^{-1}$ over the axion mass range from 24.7 to $29.1 \mu \mathrm{eV}$ at the $95 \%$ confidence level.

\section{Experiment}

We refer to this axion dark matter search as ACTION for "Axion haloscopes at CAPP with ToroIdal resONators." The ACTION experiment constitutes a tunable copper toroidal cavity, toroidal coils which provide a static magnetic field, and a typical heterodyne receiver chain. The experiment was conducted at room temperature. As shown in Figure 1, $R$ is the distance from the center

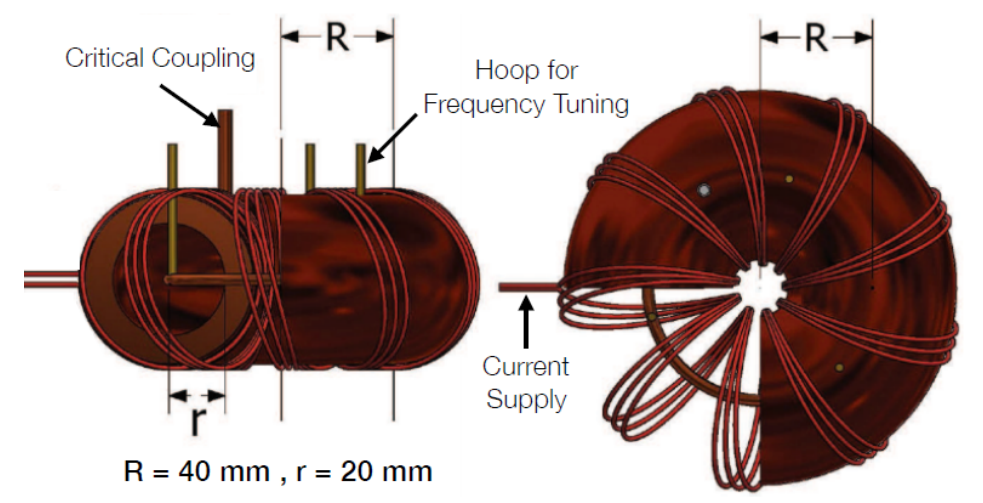

Figure 1: Lateral (Left) and top (right) views of the toroidal cavity, frequency tuning system, and toroidal coils whose dimension are given in the text. Note that it is a cut-away view to show up details of the system.

of the torus to the center of the tube and $r$ is the radius of the tube. Our cavity tube's $R$ and $r$ are 40 and $20 \mathrm{~mm}$, respectively, and the cavity thickness is $10 \mathrm{~mm}$. The frequency tuning system can be moved up and down by the piezo actuator. As a result, it provides a resonance frequency range of about $6 \sim 8 \mathrm{GHz}$.

A static magnetic field was provided by a $1.6 \mathrm{~mm}$ diameter copper wire ramped up to $20 \mathrm{~A}$ with three winding turns, as shown in Fig. 1. Figure 2 shows good agreement between measurement with a Hall probe and a simulation [6] of the magnetic field induced by the coils. The $B_{\text {avg }}$ from the magnetic field map provided by the simulation turns out to be $32 \mathrm{G}$. No fringe magnetic fields are outside of an ideal toroidal magnet, and as shown in Fig. 2, the magnetic field outside of our 


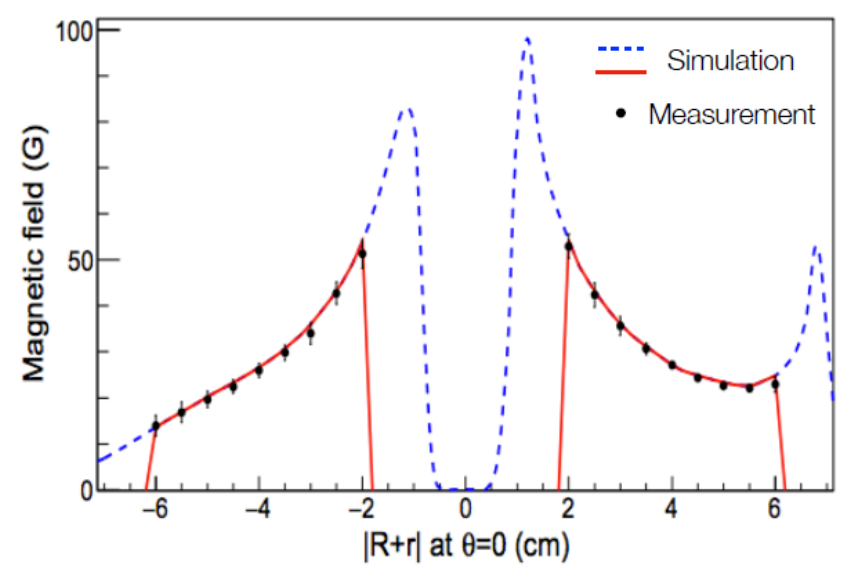

Figure 2: Magnetic field as a function of radial position $|R+r|$ at $\theta=0$. Dashed blue lines are obtained from the finite element method and correspond to the toroidal cavity system, and solid red line correspond to the cavity tube. Dots with error bars are measurement values. The results at positive $R+r$ are along a coil, while those at negative $R+r$ are between two neighboring coils.

toroidal magnet system also drops drastically, as expected.

Our receiver chain consists of a single data acquisition channel which is analogous to that adopted in ADMX [7] except for the cryogenic parts. Power from the cavity goes through RF components, is then measured by a spectrum analyzer. Cavity associates, $v$ and $Q_{L}$ are measured with a network analyzer by toggling microwave switches. The gain and noise temperature of the chain were measured to be about $35 \mathrm{~dB}$ and $400 \mathrm{~K}$ taking into account all the attenuation in the chain, for the frequency range from 6 to $7 \mathrm{GHz}$, as reported in Ref. [8].

\section{Conclusion}

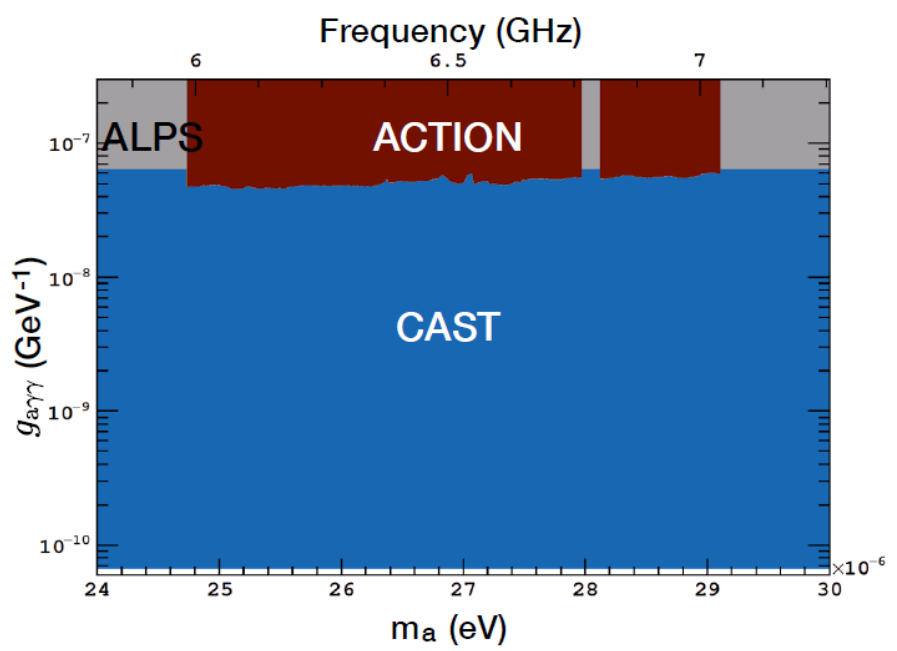

Figure 3: Excluded parameter space at the $95 \%$ C.L. by this experiment together previous results from ALPS [9] and CAST [10]. No limits are set from 6.77 to $6.80 \mathrm{GHz}$ due to with a quasi-TE mode in that frequency region [8].

Figure 3 shows the excluded parameter space at a 95\% C.L. by the simplified ACTION experiment. We demonstrate an axion haloscope with toroidal geometry and our result supersedes the pre-existing exclusion limits reported by ALPS [9] in the relevant mass ranges.

The goal of this experiment was a demonstration of an axion haloscope with toroidal geometry. We expect to reach of sensitivity of the KSVZ [11] and DFSZ [12] models by using larger-scale toroidal geometries with cooling in the future, as shown in Ref. [8]. This work was supported by IBS-R017-D1-2017-a00. 


\section{References}

[1] P. A. R. Ade et al. (Planck Collaboration), Astron. Astrophys. 594, A13 (2016).

[2] S. Weinberg, Phys. Rev. Lett. 40, 223 (1978); F. Wilczek, Phys. Rev. Lett. 40, 279 (1978).

[3] J. Preskill, M. B. Wise, and F. Wilczek, Phys. Lett. B 120, 127 (1983); L. F. Abbott and P. Sikivie, Phys. Lett. B 120, 133 (1983); M. Dine and W. Fischler, Phys. Lett. B 120, 137 (1983).

[4] John Ellis and K. A. Olive, Phys. Lett. B 193, 525 (1987); Georg Raffelt and David Seckel, Phys. Rev. Lett. 60, 1793 (1988); Michael S. Turner, Phys. Rev. Lett. 60, 1797 (1988); Hans-Thomas Janka, Wolfgang Keil, Georg Raffelt, and David Seckel, Phys. Rev. Lett. 76, 2621 (1996); Wolfgang Keil, Hans-Thomas Janka, David N. Schramm, Günter Sigl, Michael S. Turner, and John Ellis, Phys. Rev. D 56, 2419 (1997).

[5] P. Sikivie, Phys. Rev. Lett. 51, 1415 (1983); Phys. Rev. D 32, 2988 (1985).

[6] CST EM Studio, CST-Computer Simulation Technology.

https://www. cst.com

[7] H. Peng et al., Nucl. Instrum. Methods Phys. Res., Sect. A 444, 569 (2000).

[8] J. Choi, H. Themann, M. J. Lee, B. R. Ko, and Y. K. Semertzidis, Phys. Rev. D 96, 061102 (2017).

[9] K. Ehret, M. Frede, S. Ghazaryan, M. Hildebrandt, E. A. Knabbe, D. Kracht, A. Lindner, J. List, T. Meier, N. Meyer, D. Notz, J. Redondo, A. Ringwald, G. Wiedemann, and B. Willke, Phys. Lett. B 689, 149 (2010).

[10] V. Anastassopoulos et al. (CAST Collaboration), Nature Physics 13, 584-590 (2017).

[11] J. E. Kim, Phys. Rev. Lett. 43, 103 (1979); M. A. Shifmann, A. I. Vainshtein, and V. I. Zakharov, Nucl. Phys. B 166, 493 (1980).

[12] A. R. Zhitnitskii, Yad. Fiz. 31, 497 (1980) [Sov. J. Nucl. Phys. 31, 260 (1980)]; M. Dine, W. Fischler, and M. Srednicki, Phys. Lett. B 140, 199 (1981). 